\title{
Two-Dimensional Free Space Mesh Generation for Cloud-to-Ground Lightning Simulation using Finite Difference Method
}

\author{
N. Kajaluxshy and S. Thirukumaran* \\ Department of Physical Science, Vavuniya Campus, University of Jaffna
}

\begin{abstract}
The natural lightning environment is a consequence of the interaction of the lightning flash with the aircraft's body. The aircraft can, therefore, be a part of the natural lightning discharge process. Hence, the aircraft becomes exposed by direct effects to the aircraft's body and induced electromagnetic fields to the avionics held within the aircraft due to high power and short time transient. In order to study the expected electric currents and voltages produced over the surface of the aircraft, good knowledge of the waveforms, rates of rise of currents and the voltages produced by direct lightning strikes are required.
\end{abstract}

The flash of the Cloud-to-Ground lightning is represented as a wave equation which carries the parameters of current and potential of the flash. The objective is to identify the potential flow and electric field distribution along the aircraft conductor represented in the finite difference mesh under the thundercloud of $50 \mathrm{MV}$ in $1000 \mathrm{~m}$ height from the ground. A two-dimensional mesh is generated in free-space using finite difference method for the simulation and the lightning wave is presumed to be traversed in free-space where no gravitational and electromagnetic fields exist and all the boundary conditions are applied. The simulation results are comparatively significant and very useful for studying lightning impact on the aerial vehicles struck by the cloud-to-ground lightning.

Keywords: Finite difference method, two-dimensional mesh, cloudto-ground lightning

\footnotetext{
* Correspondence should be addressed to S. Thirukumaran, Department of Physical Science, Vavuniya Campus, University of Jaffna, Sri Lanka. (Email : thirukumaranvks@yahoo.com)
} 


\section{Introduction}

The design of aircraft structure and its protection systems are an important area focused by the manufacturers for flight safety from lightning strike. The metal structures of the aircraft exterior skin normally minimize the lightning effects and prevent physical damage. Modern aircrafts made by the material of carbon fibre or composite materials, being light in weight, can make the airplane fly faster. But the electric shielding effectiveness of carbon fibre is worse than metal materials. The study of electromagnetic threat due to lightning strikes is important for flight safety and restructuring the aircraft design and its electronic devices to mitigate lightning effects.

For the simulation, the lightning wave is presumed to be traversed in free-space where no gravitational and electromagnetic fields exist and all the boundary conditions are applied. To solve partial differential equation, a number of boundary conditions must be imposed. Numerical techniques are necessary for implementing boundary conditions practically.

This paper presents how a two-dimensional mesh is generated using Finite Difference Method for producing the potential distribution in the free-space due to the Cloud-to-Ground lightning flash. Finite difference method (FDM) is an important technique in the field of computations. It is a simple technique for real-time physical processes and developed for solving the wave equation (Thirukumran et al., 2013; Hoole \& Hoole, 2011; Hoole, 1993).

The electric field can be determined by finding the maximum rate and direction of spatial change of potential field. The magnitude of the electric field is largest when the derivatives of the potential are maximum. Electric fields can be computed using various methods with different precision. Electric potential V over any region depends on the ( $\mathrm{x}$ and $\mathrm{y}$ ) coordinates and its derivatives.

The objective is to find the potential flow at each grid point and represent it graphically. Another objective is to find the potential distribution of a one-dimensional vertical conductor under lightning scenario and to find the electric field distribution around the one- 
dimensional vertical conductor in the free-space under the lightning thundercloud.

\section{Literature Review}

\section{Lightning Phenomena}

Lightning is an unexpected electrostatic discharge during an electric storm between electrically charged regions of a cloud (called intracloud lightning), between that cloud and another cloud (CC lightning), or between a cloud and the ground (CG lightning) or between ground and the cloud (GC lightning). Lightning is always accompanied by the sound of thunder. Distant lightning may be seen by human eye but may be too far away for the thunder to be heard (Uman, 1984). Many factors affect the frequency, distribution, strength and physical properties of a typical lightning flash in a particular region of the world. These factors include ground elevation, latitude, prevailing wind currents, relative humidity, proximity to warm and cold bodies of water, etc. Lightning can damage or destroy them.

This paper only considers the Cloud-to-ground lightning flash because Cloud-to-Ground lightning is the most studied and best understood of the four types, even though intra-cloud, ground-tocloud and cloud-to-cloud are more common types of lightning (Hoole et al., 2014; Uman, 1984).

\section{Cloud-to-Ground Lightning}

Cloud-to-ground lightning is a lightning discharge between a thundercloud and the ground. Since the base of a thunderstorm is usually negatively charged, negative electric charges in the cloud base (thunderstorm) travel from cloud level to ground. Cloud to Ground (CG) lightning flash primarily originates in the thundercloud and terminates on a physical object that is called as Earth surface. The Cloud-to-Ground flash emanates at the bottom part of the thundercloud and travels to ground. When it connects with the ground, a return stroke wave is produced and this wave travels from ground to cloud as illustrated in Figure 1 (Thirukumran et al., 2013). 


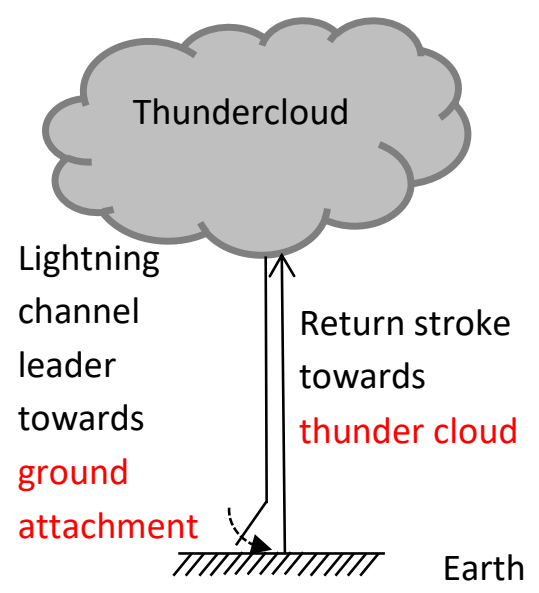

Figure 1. Cloud-to-ground flash from thunder cloud base to ground level

\section{Mesh Generation}

Cloud to Ground vertical lightning scenario is simulated by generating a two-dimensional mesh in free-space using finite difference method. The two-dimensional mesh is formulated using equal number of rows and columns. A structured mesh in two dimensions is most often simply a square grid deformed by some coordinate transformation (Hoole \& Hoole, 2011; Hoole \& Hoole, 1993). Each node of the mesh, except at the boundaries, has an isomorphic local neighbourhood. Structured meshes offer certain advantages over unstructured. They are simpler and also more convenient for use in the simpler finite difference methods (Causon \& Mingham, 2010).

\section{Finite Difference Method}

Finite difference method has been applied in an enormous number of numerical computations for a variety of problems in time domain. Finite difference method is developed to solve the wave equation (Causon \& Mingham, 2010). 


\section{Lightning Potential Wave Equation}

The wave equation for potential $\mathrm{V}$ traveling in free space is given by,

$$
\frac{\partial^{2} \mathrm{~V}(\mathrm{z}, \mathrm{t})}{\partial \mathrm{z}^{2}}-\mathrm{RC} \frac{\partial \mathrm{V}(\mathrm{z}, \mathrm{t})}{\partial \mathrm{t}}-\mathrm{LC} \frac{\partial^{2} \mathrm{~V}(\mathrm{z}, \mathrm{t})}{\partial \mathrm{t}^{2}}=0
$$

where $\mathrm{V}$ is the potential while $\mathrm{R}, \mathrm{L}$ and $\mathrm{C}$ are the resistance per unit length in Ohms, inductance per unit length in Henry and capacitance per unit length in Farad, respectively (Thirukumran et al., 2013; Hoole, 1993].

\section{Lightning Effects on the Aircraft}

The standard lightning environment is comprised of individual current and voltage waveforms, which represent the important characteristics of the natural lightning flashes. The metal aircraft or the conductive parts of the aircraft becomes part of the lightning current (Figure 2). If a conductor carries excess charge, the excess is distributed over the surface of the conductor like aircraft.

Lightning strikes cause direct and indirect damages to aircraft during take-off or landing under thundercloud. Direct effects are physical damage which usually include high voltage and current related damage to metallic or composite structures. Indirect effects are malfunctions, either temporary or permanent, that affect avionics and electrical systems (Gabrielson, 1982). The aircraft designers use analysis, laboratory measurement, and full-scale simulation to develop and demonstrate the immunity of the final design to the effects of the anticipated environment. 


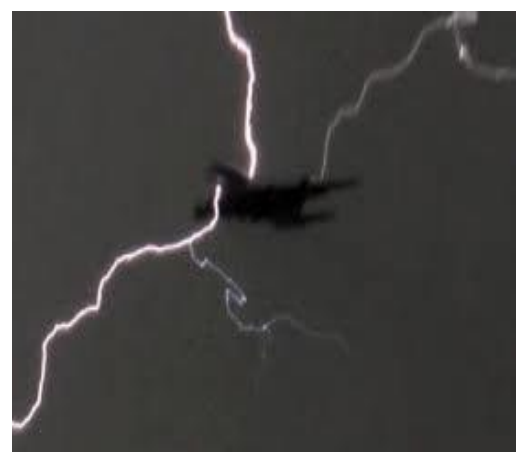

Figure 2. Lightning flash incident, Heathrow Airport, May 2011 (Neville, 2011).

There are four important factors which standardize the lightning waveforms significantly used to test the aircraft (Dunbar, 1983; RTCA/DO-160D, 2002):

1. initial stroke with peak amplitude of about $200 \mathrm{kA}$ with action integral of $2 \times 10^{6} \mathrm{~A}^{2}$-seconds in $500 \mu \mathrm{s}$,

2. intermediate average current amplitude of about $2 \mathrm{kA}$ with maximum charge transfer of about $10 \mathrm{C}$,

3. Continuing current of about $200 \mathrm{~A}$ to $800 \mathrm{~A}$ with large charge transfer of about $200 \mathrm{C}$, and

4. restrike peak current on the aircraft body of about $100 \mathrm{kA}$ with action integral of about $25 \times 10^{4} \mathrm{~A}^{2}$-s in $500 \mu \mathrm{s}$.

Accordingly, the metallic parts of the aircrafts carry lightning induced current and produce an electric field which could damage the aircraft surface and navigation system of the aircraft adversely.

\section{Electric Field}

A fundamental equation for the electric field is Laplace's equation or Poisson's equation, perhaps the simplest among many partial differential equations that express physical phenomena among various numerical calculation methods. The Finite Difference Method is very unique as it is applied exclusively to electric field calculations. This paper is based on the Finite Difference Method. 
The Finite Difference Method is not capable of calculating electric field directly at different points on the proposed region. When the potentials of the nodes are obtained, a numerical derivative evaluation technique is used to calculate the electric field intensity (Faiz \& Ojaghi, 2002). Relationship between Potential and Electric field is represented by an equation. Here, a gradient operator is used to find the electric field.

The equation is as below:

$$
E=-\nabla V
$$

where, the negative sign indicates that the field is pointing in the direction of decreasing potential, Electric field is $\mathrm{E}$ and Potential is V.

$$
\nabla \mathrm{V}=-\int \vec{E} \cdot \overrightarrow{d r}
$$

In Cartesian coordinates,

$$
\begin{aligned}
& E=E_{x}+E_{y} \text { and } \\
& d r=d x \hat{\imath}+d y \hat{\jmath} \\
& d V=\left(E_{x}+E_{y}\right) \cdot(d x \hat{\imath}+d y \hat{\jmath})=E_{x} d x+E_{y} d y
\end{aligned}
$$

which implies,

$$
\begin{aligned}
& E_{x}=-\frac{\partial V}{\partial x}, \\
& E_{y}=-\frac{\partial V}{\partial y}
\end{aligned}
$$

By introducing a differential quantity called the gradient operator,

$$
\nabla \equiv \frac{\partial}{\partial \mathrm{x}} \hat{\imath}+\frac{\partial}{\partial y} \hat{\jmath}
$$

The Electric field can be written as, 


$$
E=E_{x} \hat{\imath}+E_{y} \hat{\jmath}=-\left(\frac{\partial V}{\partial x} \hat{\imath}+\frac{\partial V}{\partial y} \hat{\jmath}\right)=-\left(\frac{\partial}{\partial x} \hat{\imath}+\frac{\partial}{\partial y} \hat{\jmath}\right) V=-\nabla V
$$

Notice that $\nabla$ operates on a scalar quantity (electric potential) and results in a vector quantity (electric field). Mathematically, we can think of $\mathrm{E}$ as the negative of the gradient of the electric potential V. Physically, the negative sign implies that if $\mathrm{V}$ increases as a positive charge moves along some direction, there is a component of $\vec{E}$ in the opposite direction.

Let, two-dimensional problem in which the potential does not vary with the $z$ coordinate. The unknown values of the potential at five adjacent points are indicated as $\mathrm{V}_{0}, \mathrm{~V}_{1}, \mathrm{~V}_{2}, \mathrm{~V}_{3}$ and $\mathrm{V}_{4}$ (Figure 3). If the region is charge-free and contains a homogeneous dielectric, then $\vec{\nabla} . \vec{E}=0$, from which we have, in two-dimensions

$$
\frac{\partial E_{x}}{\partial x}+\frac{\partial E_{y}}{\partial y}=0
$$

But the gradient operation gives

$$
\begin{aligned}
& \mathrm{E}_{\mathrm{x}}=-\frac{\partial V}{\partial x}, \\
& \mathrm{E}_{\mathrm{y}}=-\frac{\partial V}{\partial y}
\end{aligned}
$$

from which we obtain

$$
\frac{\partial^{2} \mathrm{~V}}{\partial \mathrm{x}^{2}}+\frac{\partial^{2} \mathrm{~V}}{\partial \mathrm{y}^{2}}=0
$$

This is simply given by the Poisson's equation $\nabla^{2} V=0$.

Approximate values for these partial derivatives may be obtained in terms of the assumed potentials, or

$$
\begin{aligned}
& \left.\frac{\partial V}{\partial x}\right|_{\mathrm{a}}=\left(\mathrm{V}_{1}-\mathrm{V}_{0}\right) / \mathrm{h} \\
& \frac{\partial V}{\partial x} \mathrm{I}_{\mathrm{c}}=\left(\mathrm{V}_{0}-\mathrm{V}_{3}\right) / \mathrm{h}
\end{aligned}
$$




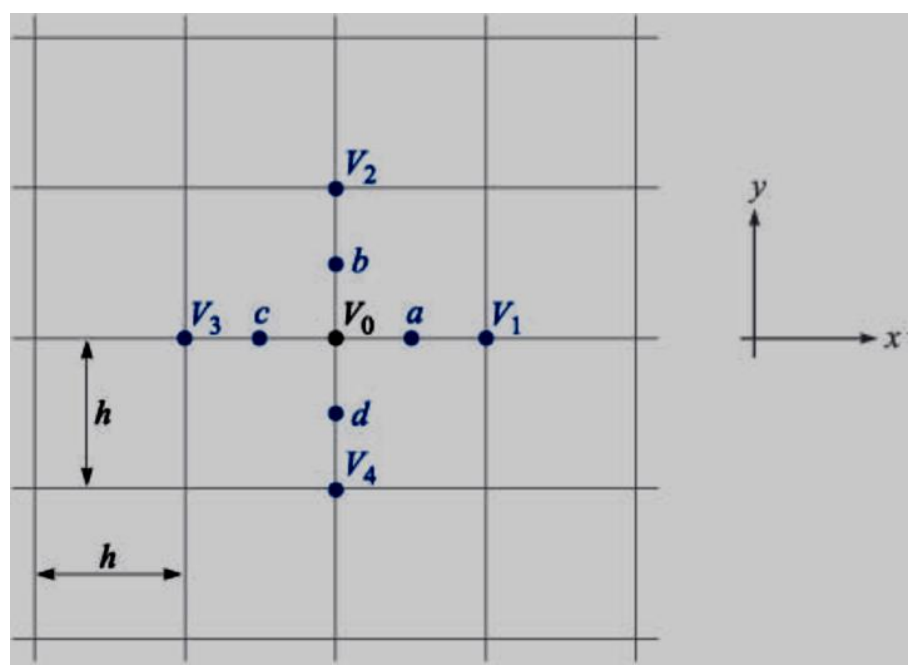

Figure 3. Squares of length $h$ on a side, the potential $V_{0}$ is approximately equal to the average of the potentials at the four neighbouring nodes.

$$
\begin{aligned}
& \left.\frac{\partial^{2} \mathrm{~V}}{\partial \mathrm{x}^{2}}\right|_{0}=\frac{\left.\frac{\partial \mathrm{V}}{\partial \mathrm{x}}\right|_{\mathrm{a}}-\left.\frac{\partial \mathrm{V}}{\partial \mathrm{x}}\right|_{\mathrm{c}}}{\mathrm{h}^{2}}=\left(\mathrm{V}_{1}-\mathrm{V}_{0}-\mathrm{V}_{0}+\mathrm{V}_{3}\right) / \mathrm{h}^{2} \\
& \left.\frac{\partial^{2} \mathrm{~V}}{\partial \mathrm{y}^{2}}\right|_{0}=\frac{\left.\frac{\partial \mathrm{V}}{\partial \mathrm{y}}\right|_{\mathrm{b}}-\left.\frac{\partial \mathrm{V}}{\partial \mathrm{y}}\right|_{\mathrm{d}}}{\mathrm{h}^{2}}=\left(\mathrm{V}_{2}-\mathrm{V}_{0}-\mathrm{V}_{0}+\mathrm{V}_{4}\right) / \mathrm{h}^{2} \\
& \frac{\partial^{2} \mathrm{~V}}{\partial \mathrm{x}^{2}}+\frac{\partial^{2} \mathrm{~V}}{\partial \mathrm{y}^{2}}=\left(\mathrm{V}_{1}+\mathrm{V}_{2}+\mathrm{V}_{3}+\mathrm{V}_{4}-4 * \mathrm{~V}_{0}\right) / \mathrm{h}^{2}=0 \\
& \mathrm{~V}_{0}=\left(\mathrm{V}_{1}+\mathrm{V}_{2}+\mathrm{V}_{3}+\mathrm{V}_{4}\right) / 4
\end{aligned}
$$

The expression becomes exact as $\mathrm{h}$ approaches zero. It is intuitively correct, telling us that the potential is the average of the potential at the four neighbouring points. The iterative method is merely used to determine the potential at the corner of every square subdivision in turn, and then the process is repeated over the entire region as many times as is necessary until the values no longer change (Hayt \& Buck, 2014). 


\section{Mesh Generation for Lightning Simulation}

\section{Mesh Generation}

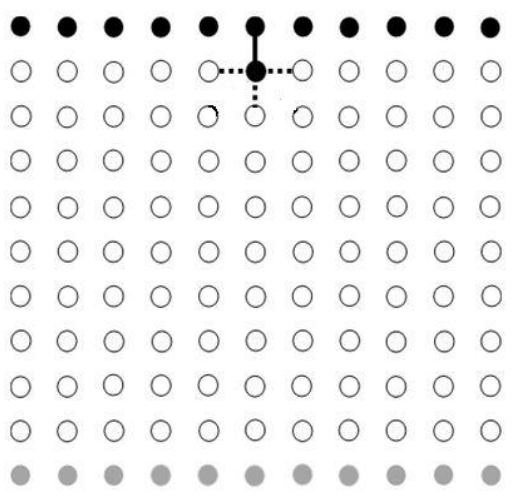

Figure 4. Initial Cloud-to-ground flash distribution in the mesh

Figure 4 shows a sample square mesh. It can be divided into equal size of rows and columns. It produces lightning discharge patterns in two-dimension. Cloud base and ground level in the mesh are indicated by black and Grey circles respectively. Other grid nodes are indicated by white circles (Perera \& Sonnadara, 2012). All the grid points in the two-dimensional mesh in the height from ground level to cloud level will be processed to identify the potential flow of the Cloud-to-Ground lightning flash. The lightning voltage is distributed at each grid point in the free-space. All grid point voltage depends on its four neighbouring node voltage.

Each node point has an electric potential value $\mathrm{V}$ associated with it. Initially the potential of the node points in the upper boundary (cloud base) of the mesh are fixed to $50 \mathrm{MV}$, while node points in the lower boundary (ground level) are fixed to OV.

\section{Initial Environment Potential Distribution}

The top row (first row) of the mesh maintains the cloud voltage (50 MV) and bottom row (last row) of the mesh maintains the ground voltage $(0 \mathrm{~V})$. Except the cloud and ground level grid points, voltage (white circle nodes) is calculated based on the potential difference 
between the cloud voltage and ground voltage and the number of rows.

$\mathrm{D}=\mathrm{NC}$ or number of grid points in the vertical line -1

$\mathrm{C}=\mathrm{CV} / \mathrm{D}$

$\mathrm{CRV}=\mathrm{PRV}-\mathrm{C}$

where,

$$
\begin{aligned}
& \mathrm{D}=\text { Distance } \\
& \mathrm{NC}=\text { Number of columns } \\
& \mathrm{C}=\text { Change or voltage difference } \\
& \mathrm{CV}=\text { Cloud voltage } \\
& \mathrm{CRV}=\text { Current row voltage } \\
& \mathrm{PRV}=\text { Previous row voltage }
\end{aligned}
$$

Here, the change means voltage difference between each horizontal line.

We can find voltage of other charge free nodes in the mesh initially using the equation (23).

\section{Potential Distribution using Finite Difference Method}

Finite difference method can be used to calculate the potential at the nodes. The accuracy of the numerical results depends on the size of the computational mesh. When we start to find the potential at each node of the mesh using finite difference method we find the potential from top to bottom row wise and left to right in column wise. We start at position $(2,2)$ go along the whole row, repeat for the next row etc., and eventually end at position (N-1, N-1). Here, $\Delta \mathrm{x}=\Delta \mathrm{y}$. 


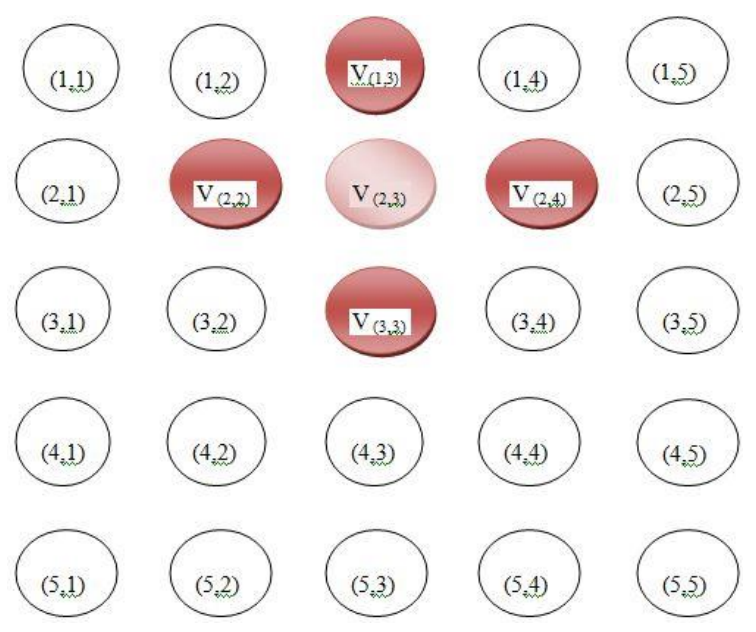

Figure 5. $(5 \times 5)$ matrix (or mesh) potential distribution calculated by finite difference method

This is the general form of finding the potential distribution at each node using finite difference method.

$$
\mathrm{V}_{\mathrm{i}, \mathrm{j}}=\left(\mathrm{V}_{\mathrm{i}-1, \mathrm{j}}+\mathrm{V}_{\mathrm{i}, \mathrm{j}-1}+\mathrm{V}_{\mathrm{i}, \mathrm{j}+1}+\mathrm{V}_{\mathrm{i}+1, \mathrm{j}}\right) / 4
$$

For example, in Figure 5, the potential at node $(2,3)$ is calculated by:

$$
\mathrm{V}_{2,3}=\left(\mathrm{V}_{1,3}+\mathrm{V}_{2,2}+\mathrm{V}_{2,4}+\mathrm{V}_{3,3}\right) / 4
$$

The last interior node in the mesh is $(\mathrm{N}-1),(\mathrm{N}-1), \mathrm{N}=\mathrm{n}$

If we find the potential at node $(n-1, n-1)$ is:

$$
\mathrm{V}_{\mathrm{n}-1, \mathrm{n}-1}=\left(\mathrm{V}_{\mathrm{n}-2, \mathrm{n}-1}+\mathrm{V}_{\mathrm{n}-1, \mathrm{n}-2}+\mathrm{V}_{\mathrm{n}-1, \mathrm{n}}+\mathrm{V}_{\mathrm{n}, \mathrm{n}-1}\right) / 4
$$

The centre node potential in the mesh is:

$$
\mathrm{V}_{\mathrm{C}}=\left(\mathrm{V}_{\mathrm{T}}+\mathrm{V}_{\mathrm{B}}+\mathrm{V}_{\mathrm{L}}+\mathrm{V}_{\mathrm{R}}\right) / 4
$$

where,

$$
\begin{aligned}
& V_{C}=\text { Voltage of the Centre node } \\
& V_{T}=\text { Voltage of the Top node } \\
& V_{B}=\text { Voltage of the Bottom node }
\end{aligned}
$$




$$
\begin{aligned}
& V_{L}=\text { Voltage of the Left node } \\
& V_{R}=\text { Voltage of the Right node }
\end{aligned}
$$

In this way we can find voltage of all nodes in the mesh using Finite difference method.

\section{Potential Distribution of a 1-D Vertical Conductor}

This paper presents another objective to find the potential distribution and the electric field distribution along the onedimensional vertical conductor (Figure 6) and other grid points in the mesh using Finite difference method.

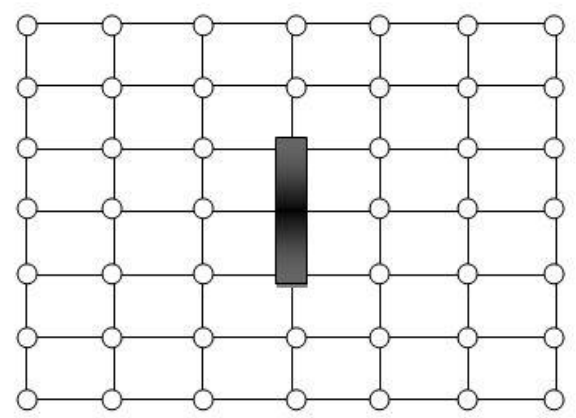

Figure 6. Mesh contains equal number of rows and columns, after we include one vertical conductor in the middle of the mesh.

Now all the grid points in the mesh contain the potential distribution. The grid points along the one-dimensional conductor also contain the potential value, then find average of those values and assigned this average voltage into all the conductor points to maintain equal conductivity. This average voltage is the initial potential along the conductor points.

All the conductor points have the same initial potential value and then we start to iterate using Finite difference method. It is useful for finding the voltage along the conductor and other grid points in the mesh. Iteration started from top to bottom in the row wise and 
left to right in the column wise. If we find the first position of the conductor voltage and assigned this voltage into all other conductor points. Then move to the next node in the mesh. Every time we find the conductor point voltage in the mesh and this voltage is assigned along the conductor points. Last point on the conductor voltage is the final voltage along the conductor points.

\section{Electric Field Distribution around the Conductor}

We find the Vertical field in the y-direction (vertical). This value depends on potential of the row nodes in the mesh.

$$
\text { Vertical field }(\mathrm{V})=(\mathrm{Vy} 1-\mathrm{Vy} 2) / \mathrm{dy}
$$

Similarly, we can find the Horizontal field in the $\mathrm{x}$-direction (horizontal). This value depends on potential of the column nodes in the mesh.

$$
\text { Horizontal field }(\mathrm{H})=(\mathrm{Vx} 2-\mathrm{Vx} 1) / \mathrm{dx}
$$

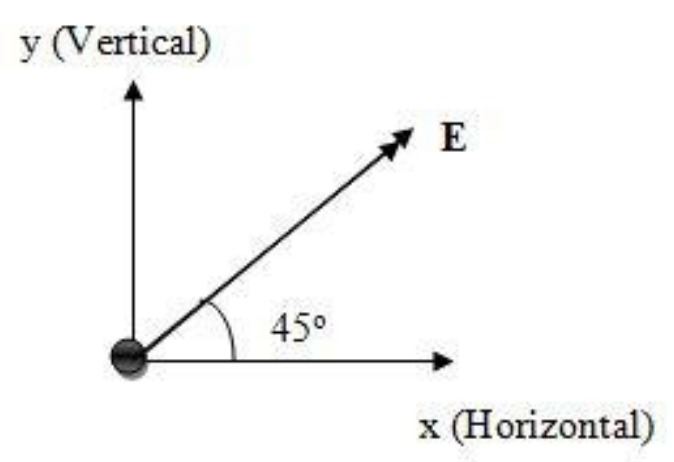

Figure 7. Electric field in the xy-direction and Total electric field is E.

The first component of the equation (11) is in the x-direction. The second component of it is in the y-direction. If we contain the potential of both $\mathrm{x}$ and $\mathrm{y}$-direction, then we can find the electric field 
around the conductor. Here, equal number of rows and columns are involved so, the degree is $45^{\circ}$ (Figure 7).

$(\text { Electrical field })^{2}=(\text { Vertical field })^{2}+(\text { Horizontal field })^{2}(30)$

$$
\begin{aligned}
& E^{2}=V^{2}+H^{2} \\
& E=\sqrt{V^{2}+H^{2}}
\end{aligned}
$$

$\mathrm{V}$ and $\mathrm{H}$ could be calculated using the equations (28) and (29) respectively. If we know the values of $\mathrm{V}$ and $\mathrm{H}$ then we can find the value of $\mathrm{E}$.

\section{Results}

This paper provides a practical overview of numerical solutions of the Cloud-to-Ground lightning wave equation using finite difference method. For the simulation, the inputs are cloud voltage of $-50 \mathrm{MV}$ and ground voltage of OV. A negative value is used for cloud voltage since negative charges are accumulated at the bottom of the cloud region from where the lightning leader propagates towards ground. Furthermore, the stability and the accuracy of finite difference time domain method (FDTD) are ensured by numerical computation to identify the lightning characteristics (Hoole \& Hoole, 2011).

Figure 8 shows, the potential distribution along the 1-D vertical conductor located below the thundercloud in three different heights above the ground. In the figures, high voltage appeared closer to the cloud level in blue and low potential values are indicated closer to the ground level in red. The potential distribution along the vertical conductor closer to the cloud level (Figure 8(a)), middle level (Figure 8(b)) and closer to the ground level (Figure 8(c)) are shown. The potential of the conductor remains constant in each of the cases. 


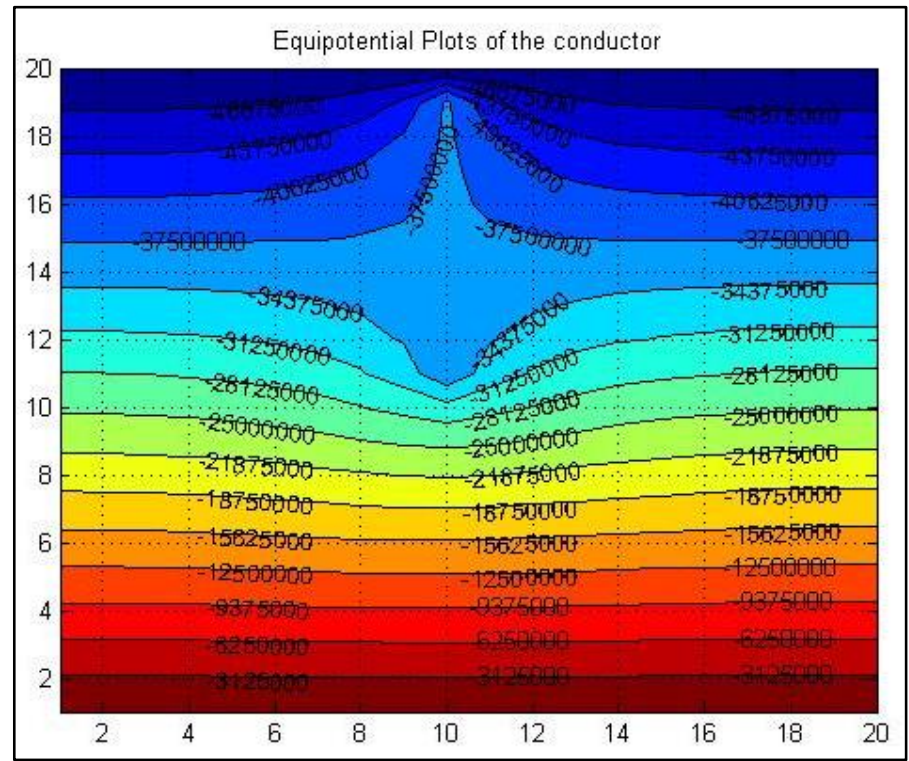

Figure 8(a). Potential distribution of a 1-D vertical conductor closer to the cloud level

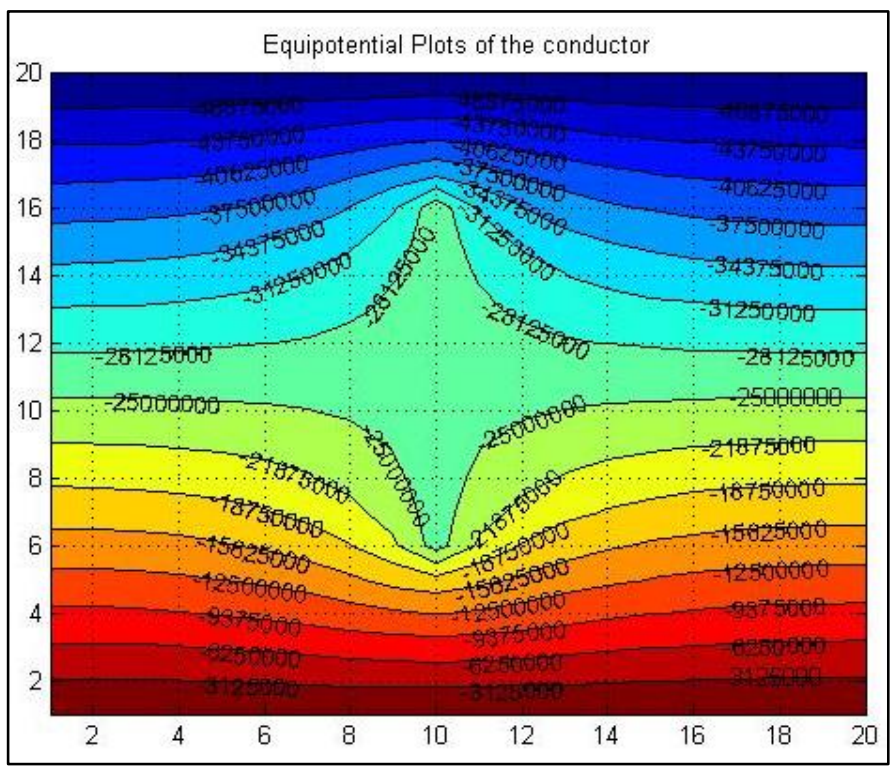

Figure 8(b). Potential distribution of a 1-D vertical conductor at middle 


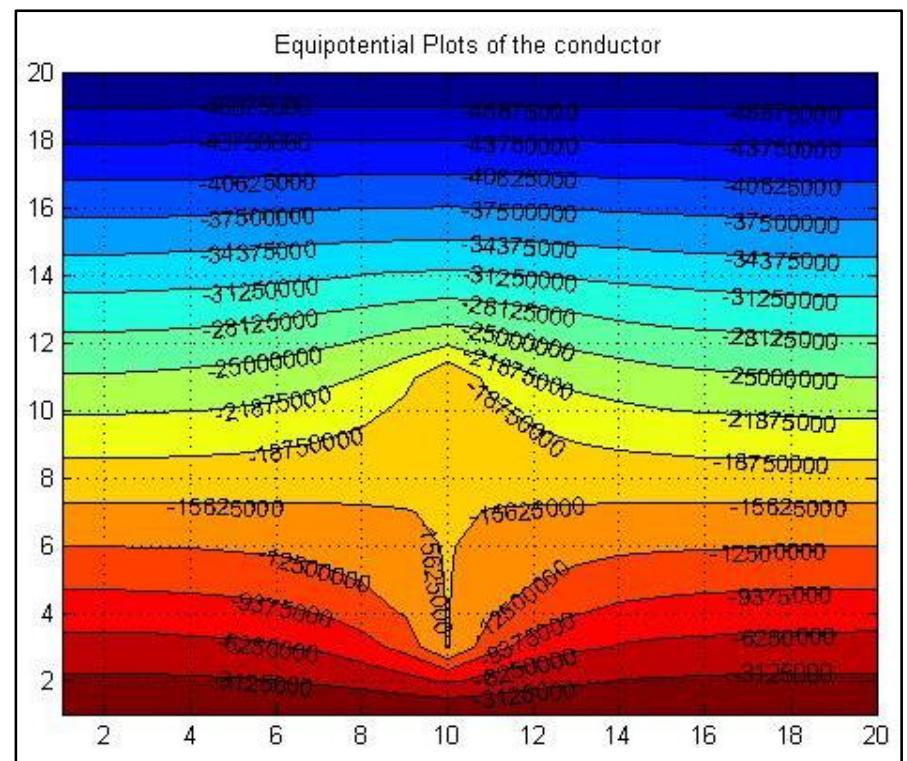

Figure 8(c). Potential distribution of a 1-D vertical conductor closer to the ground level

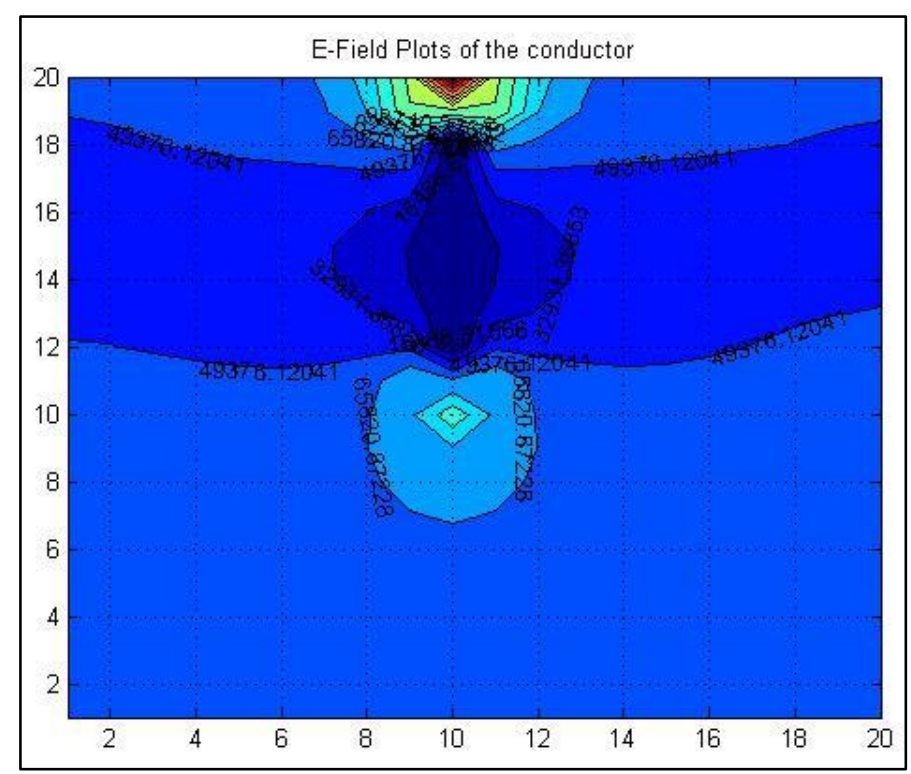

Figure 9(a). Electric field distribution around the 1-D vertical conductor closer to the cloud level 


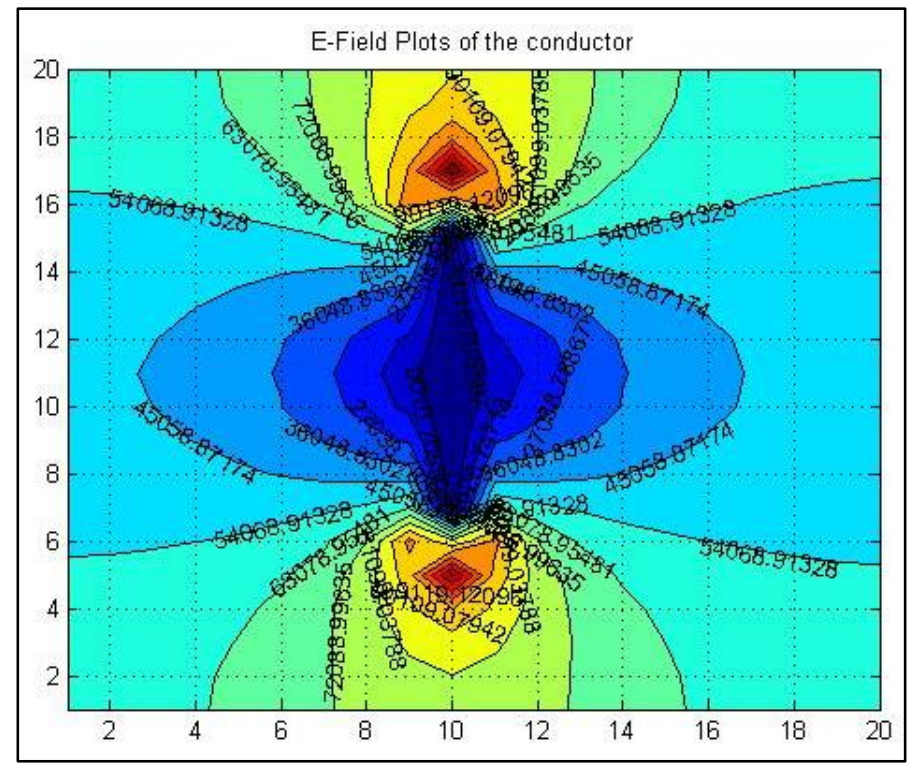

Figure 9(b). Electric field distribution around the 1-D vertical conductor at middle

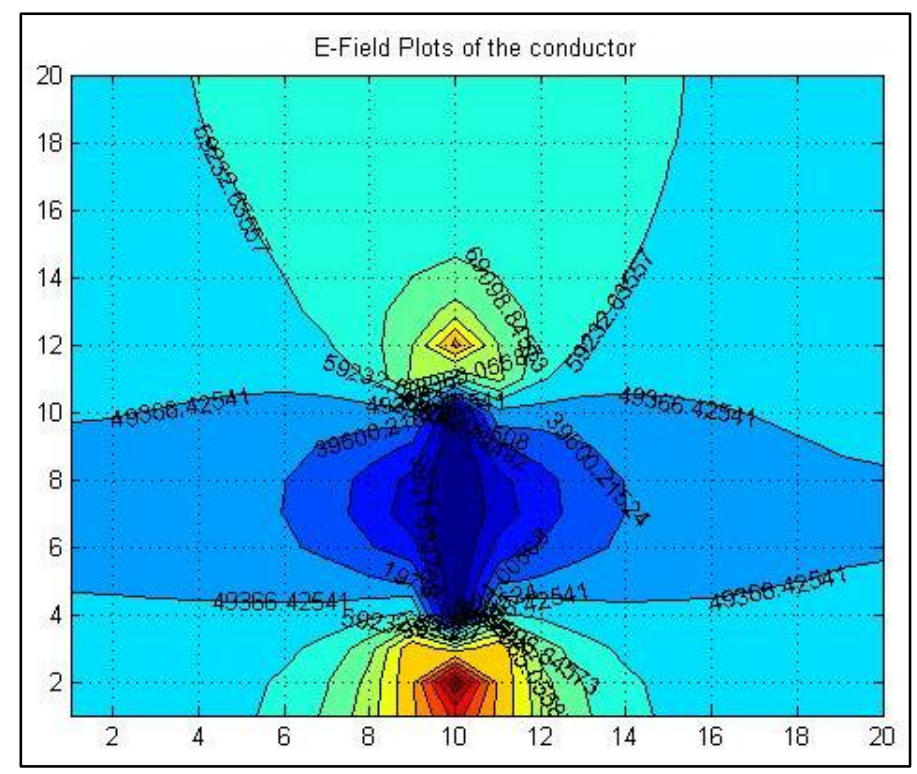

Figure 9(c). Electric field distribution around the 1-D vertical conductor closer to the ground level 
Figure 9 shows the initial simulation results for the electric field distribution around the one-dimensional conductor located in three different heights: closer to the cloud level (Figure 9(a)), middle level (Figure 9(b)) and closer to the ground level (Figure 9(c)) are shown under the thundercloud and above the ground. The simulation results show the electric field distribution at the end points of the conductor is significant and much higher than the electric field distribution of other points around the conductor because of the sharp edges. High electric fields are indicated by red at the endpoints. The electric field on the interior points along the conductor is zero in all three cases since the vertical potential difference is zero on the conductor. The initial simulation shows that the magnitude of radiated electric fields interacts with the aircraft resulting in adversely on the aircraft navigation systems which may cause damage to its structures.

The Figure 8 and 9 give initial simulation results for the potential and electric field distribution of a single vertical conductor under the lightning scenario. The potential and electric field distribution are to be identified for different shapes of the conductor to measure its effects. The above study would help to model the aircraft and identify the lightning effects on the aircraft surface and around it. Moreover, it is assumed that the fuselage of an aircraft being a good conductor and the effects of material properties are left for future studies.

\section{Conclusion}

The aircraft-lightning interaction under the thundercloud and above the ground is considered. This paper presents generating a twodimensional mesh in free space scenario using finite difference method for producing the potential and electric field distribution of the Cloud-to-Ground lightning flash. In the simulation, the lightning voltage was distributed numerically at each grid point and graphically represented. A significant increase of induced electric field was observed in the simulation due to the lightning stroke to aircraft conductor. The potential and electric field distribution are developed along and around the one dimensional conductor and 
other grid points in the mesh in between the thundercloud and the ground.

In this initial simulation, the voltage surges and the electric field distributions are computed and it is reported that a higher rate of rise of electric field was observed due to cloud to ground lightning flash. These measures obtained would be very useful for developing lightning impact on the aerial vehicles struck by the cloud-to-ground lightning. The work presented in this paper would also be useful for further extension of modelling the conductor like aircraft with the effects of material properties.

Furthermore, according to the evidences which show that the aircraft initiates lightning during take-off under thundercloud, the presented work could be extended to study situations where the aircraft-lightning interaction occurs in different heights while flying between subsequent lightning strikes to identify the effects on the surface of the aircraft and avionics within the aircraft.

\section{References}

Causon, D. M., \& Mingham, C. G. (2010). Introductory Finite Difference Method for PDEs, Ventus Publishing ApS. ISBN 978-87-7681-642-1.

Dunbar, W. G. (1983). HV Design Guide: Aircraft, Boeing Aerospace Company.

Faiz, J. \& Ojaghi, M. (2002). Instructive Review of Computation of Electric Fields using Different Numerical Techniques, Int. J. Engng Ed. Vol. 18(3): 344-356.

Gabrielson, B. C. (1982). Lightning Protection for Rocket.

Gromico, N. (2006). Wind Turbines and Lightning, InterNACHI. https://www.nachi.org/wind-turbines-lightning.htm (accessed 20 March 2016)

Hayt, W. H. Jr, Buck, J. A. (2014). Experimental Mapping Method, Engineering Electromagnectics, Sixth Edition. Mc Graw Hill. 
Hoole, P. R. P. (1993). Modelling The Lightning Earth Flash Return Stroke for Studying its Effects on Engineering Systems. IEEE Transactions on Magnetics, Vol. 29(2): 1839 - 1844. https://doi.org/10.1109/20.250764

Hoole, P. R. P. and S. R. H. Hoole. (2011). Stability and Accuracy of the Finite Difference Time Domain (FDTD) Method to Determine Transmission Line Traveling Wave Voltages and Currents: The Lightning Pulse. Journal of Engineering and Technology Research, Vol. 3(2): 50-53.

Hoole, P. R. P. \& Hoole, S. R. H. (2011). A Distributed Transmission Line Model of Cloud-to-Ground Lightning Return Stroke: Model Verification, Return Stroke Velocity, Unmeasured Currents and Radiated Fields. International Journal of the Physical Sciences, Vol. 6(16): 3851-3866.

Hoole, P. R. P. \& Hoole, S. R. H. (1993). Simulation of Lightning Attachment to Open Ground, Tall Towers and Aircraft. IEEE Transactions on Power Delivery, Vol. 8(2): 732-740. https://doi.org/10.1109/61.216882

Hoole, P. R. P., Thirukumaran, S., Ramiah, H., Kanesan, J. \& Hoole, S. R. H. (2014). Ground to Cloud Lightning Flash Currents and Electric Fields: Interaction with Aircraft and Production of Ionosphere Sprites. Journal of Computational Engineering, Vol. 2014, Article ID 869452, 5 pages. http://dx.doi.org/10.1155/2014/869452

Neville, S. (2011). Electrifying: The terrifying moment a jet was struck by lightning on approach to Heathrow. Available online at:

http://www.dailymail.co.uk/news/article-1386086/Jetstruck-lightning-lands-Heathrow.html\#ixzz 1dGEEkLOL (accessed 20 March 2016) 
Perera, M. D. N. \& Sonnadara, D. U. J. (2012). Fractal Nature of Simulated Lightning Channels. Sri Lankan Journal of Physics, Vol. 13(2): 09-25. http://dx.doi.org/10.4038/sljp.v13i2.5433

RTCA/DO-160D, (2002). Environmental Conditions and Test Procedure for Aircraft Equipment.

Thirukumaran, S., Hoole, P. R. P., Harikrishnan, R., Jeevan, K., Pirapaharan, K. \& Hoole, S. R. H. (2013). Aircraft-Lightning Electrodynamics using the Transmission Line Model Part I: Review of the Transmission Line Model. Progress In Electromagnetics Research M, Vol. 31: 85-101. http://dx.doi.org/10.2528/PIERM12110303

Uman, M. A. (1984). Lightning, New York, Dover.

Received: 28-03-2016 Revised: 01-07-2016 Accepted: 04-07-2016 